\title{
Las Poáceas del Parque Nacional Yanachaga-Chemillén, Oxapampa, Pasco, Perú. Parte I: Bambusoideae
}

\section{Poaceae from Yanachaga-Chemillén National Park (Oxapampa, Pasco, Perú). Part I: Bambusoideae}

\author{
María I. La-Torre ${ }^{1,2}$, Asunción Cano² y Oscar Tovar².
}

Presentado: $18 / 11 / 2003$

Aceptado: $12 / 12 / 2003$

\section{Resumen}

El presente trabajo es la primera parte de un estudio de la biodiversidad de la familia Poaceae del Parque Nacional Yanachaga-Chemillén y zonas aledañas, (10은 $35^{\prime}-10^{\circ} 10^{\prime}$ LS y $75^{\circ} 10^{\prime}-75^{\circ} 45^{\prime}$ LO, y desde los 300 hasta los $3800 \mathrm{~m}$ ). El material utilizado procedió de colectas realizadas por los autores desde 1995 a 1996 y de material depositado en el Herbario San Marcos (USM) del Museo de Historia Natural y del Missouri Botanical Garden (MO). En esta primera parte se da a conocer la diversidad de la subfamilia Bambusoideae. En el área de estudio han sido registradas para la subfamilia Bambusoideae 7 géneros y 23 especies. Entre las Bambusoideas leñosas el género Chusquea presentó el mayor número de especies (siete). El género Olyra es el mejor representado entre las Bambusoideas herbáceas con cinco especies. Se presenta una breve descripción de la subfamilia y los géneros, así como también claves para diferenciar los géneros y especies. Para cada especie se indican datos de su ecología, distribución y el material estudiado.

Palabras clave: Poáceas, Bambusoideas, Yanachaga-Chemillén, Perú.

\section{Abstract}

This work is the first part of biodiversity study of Poaceae family from Yanachaga-Chemillen National

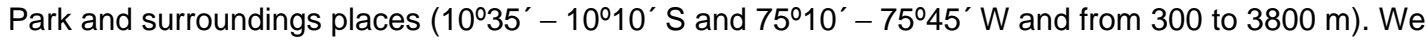
analysed the material colected for us since 1995 to 1996 and material deposited in the Herbario San Marcos of Museo de Historia Natural and Missouri Botanical Garden. This first part is dedicated to the Bambusoideae biodiversity. A total of seven genera and 23 species have been registered. In the woody bamboos group, genus Chusquea was the mayor species number (seven). The genus Olyra is the most diverse of the herbaceous bamboos with five species. Descriptions of subfamily, genera and keys for genera and species are developed. Ecology, distribution and studied material are indicated.

Keywords: Poaceae, Bambusoideae, Yanachaga-Chemillén, Perú.

\section{Introducción}

La familia Poaceae (Gramineae) esta ampliamente distribuida en el mundo, sus especies se encuentran presentes en todas las latitudes y en altitudes, desde el nivel del mar hasta por encima de los $5000 \mathrm{~m}$. Las Poaceae son una de las familias con mayor número de especies, con cerca de 750 géneros y 10000 especies en todo el mundo. En la flora perua-

${ }^{1}$ Facultad de Ciencias Naturales y Matemáticas, Universidad Nacional Federico Villarreal, Calle San Marcos, 351, Lima 21.

${ }^{2}$ Museo de Historia Natural, Universidad Nacional Mayor de San Marcos, Avda. Arenales 1256, Lima 11.

E-mail, Maria I. La-Torre: marycano_11@yahoo.com na, están representadas por alrededor de 700 especies agrupadas en 160 géneros, destacando la presencia de todas las subfamilias existentes en el mundo (Tovar, 1993). En el Perú se les encuentran en todos los pisos bioclimáticos, desde el nivel del mar hasta el piso subnival de los Andes y la llanura amazónica.

Las Poaceae son elementos florísticos importantes en la mayoría de las formaciones vegetales que ocurren en el territorio peruano (Weberbauer, 1945; Brack, 1986; Young y Valencia, 1992; Brako y Zarucchi, 1993; Cano, 1994). En el Parque Nacional YanachagaChemillén, las Poaceae, al igual que la mayo- 
ría de los taxa, no han sido estudiadas con la profundidad requerida y se desconoce su diversidad, relaciones florísticas y ecológicas. Incluso existen grandes zonas del parque no exploradas por su inaccesibilidad, tal como sucede con la mayor parte de la cordillera Yanachaga.

El objetivo del trabajo fue realizar un estudio taxonómico de la familia Poaceae del Parque Yanachaga-Chemillén y zonas aledañas, con la finalidad de contribuir al conocimiento integral de la flora de esta unidad de conservación, así como de la flora peruana en general. En esta oportunidad presentamos los resultados referentes a la Subfamilia Bambusoideae, que incluye tanto bambúes leñosos como herbáceos.

\section{Área de estudio}

El Parque Nacional Yanachaga-Chemillén está situado en el departamento de Pasco, provincia de Oxapampa y en los distritos de
Pozuzo, Huancabamba, Oxapampa y Villa Rica. Está comprendido entre las coordenadas $10^{\circ} 35^{\prime}-10^{\circ} 10^{\prime} \mathrm{S}$ y $75^{\circ} 10^{\prime}-75^{\circ} 45^{\prime} \mathrm{O}(\mathrm{Fi}-$ gura 1). El Parque fue creado el 30 de agosto de 1986 mediante Decreto Supremo No 06886-AG (Brack, 1984). El Parque protege variados ecosistemas en un rango altitudinal que va desde los 300 hasta los $4000 \mathrm{~m}$, comprendiendo selva baja, bosque premontano, bosque montano y fragmentos de pajonal de puna.

Su nombre compuesto se origina de dos vocablos, el primero, «yanachaga», proviene del quechua, cuyo significado se interpreta como «bulto negro». El segundo proviene del yanesha, idioma de los nativos de la región que le denominan «chemillén», voz que se traduce como «negro» o «quemado», aludiendo al aspecto oscuro o casi negro que toman estas montañas al atardecer de días soleados, al ser divisadas a lo lejos desde el valle (Aguilar, 1986; Pacheco et al., 1994).

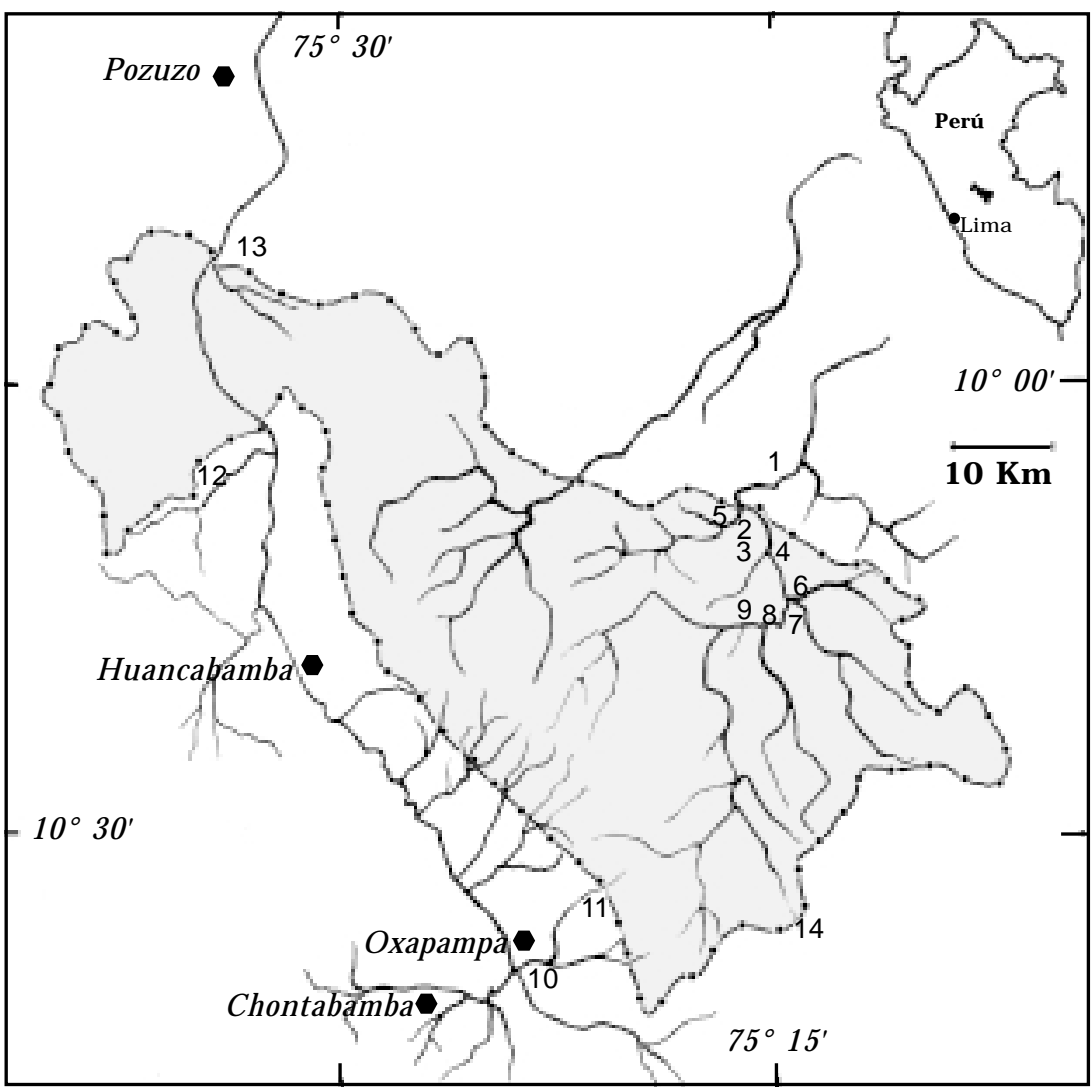

Figura 1. Mapa del área de estudio mostrando la ubicacion de las localidades de colecta. (1) Playa Caliente, (2) Estacion ecológica de Paujil, (3) Mirador, (4) Río Venado, (5) Río Paujil, (6) Río Lobo, (7) Subestacion Río Pescado, (8) Río San carlos, (9) Laguna Luna Llena, (10) Oxapampa, (11) San Alberto, (12) Santa Bárbara, (13) Quebrada Tunqui, (14) Cerro Pajonal San Gotardo 


\section{Material y métodos}

Para la realización del trabajo de campo se emplearon los materiales y técnicas usuales en las colectas botánicas (Cerrate, 1969; Womersley, 1981; Lot y Chiang, 1986). Las localidades de colecta se georeferenciaron con un GPS (Global Position System). Las expediciones de colecta y estudio se llevaron a cabo entre los años 1995-1996. Las colecciones se realizaron en diferentes altitudes desde los 300 $\mathrm{m}$ hasta los $3800 \mathrm{~m}$.

La determinación de las muestras se realizó empleando claves y descripciones publicadas en trabajos de la especialidad, como Burkart (1969), Pohl (1980), Nicora y Rugolo de Agrasar (1987), Clark y Londoño (1990, 1991), Clayton \& Renvoize (1986), Tovar (1993), Renvoize (1998). Además, se realizaron comparaciones con ejemplares del Herbario San Marcos (USM) y del Missouri Botanical Garden (MO), y también se realizaron consultas con especialistas en la familia Poaceae.

Además del material colectado por los autores se ha incluido en el estudio especímenes colectados por David N. Smith, Robin Foster, Sandra Knapp y Blanca León los que se encuentran depositados en los Herbario San Marcos (USM) del Museo de Historia Natural y en el del Missouri Botanical Garden (MO).

\section{Resultados}

\section{Carácteres Taxonómicos de la familia Poaceae}

La familia POACEAE comprende plantas herbáceas, raramente leñosas, anuales o perennes, cespitosas, rizomatosas o estoloníferas, con escasos o numerosos entrenudos; los entrenudos pueden ser sólidos o huecos, las hojas de disposición dística, con pecíolo modificado llamado vaina; la lígula se ubica entre la vaina y la lámina, ésta puede ser membranácea o pubescente, raramente ausente, a veces con lóbulos laterales llamadas aurículas; lámina foliar linear; en algunos casos aovado lanceolada, plana o enrollada; paralelinervada; en algunos grupos como las Bambusoideas, la base angosta de la lámina es llamada pseudopecíolo. Inflorescencia en el ápice de las cañas y con ramificaciones terminales o axilares, laxas o contraídas, en espiga , racimo o panoja; la espiguilla es la unidad básica de la inflorescencia y presenta una estructura muy diversa. La espiguilla consta de un eje, la raquila, que lleva en su base dos brácteas estériles llamadas glumas de diverso tamaño y consistencia; en el interior se encuentra el flósculo, que consiste de una bráctea exterior, la lema, y otra interior la pálea, esta última envuelve a la flor que puede ser hermafrodita o unisexual, con el perianto reducido a excrecencias escamosas turgentes llamadas lodículas. Se denomina también antecio al conjunto de lema y pálea sostenido por el callo, pequeño pie pungente o romo, glabro o pubescente. El ovario es unilocular con dos estigmas plumosos, estambres comúnmente tres, aunque en Pariana son numerosos. El fruto es, por lo general, un cariópside.

\section{Diversidad de la familia Poaceae en el Parque Nacional Yanachaga-Chemillén}

Se han determinado seis subfamilias, 16 tribus, 44 géneros y 86 especies (Tabla1).

Tabla 1. Número de Subfamilias, Tribus, Géneros y Especies de la familia Poaceae determinadas para el Parque Nacional YanachagaChemillén.

\begin{tabular}{lccc}
\hline Subfamilia & Tribus & Géneros & Especies \\
\hline Bambusoideae & 4 & 7 & 23 \\
Centothecoideae & 1 & 2 & 2 \\
Panicoideae & 3 & 18 & 34 \\
Arundinoideae & 1 & 2 & 3 \\
Chloridoideae & 3 & 6 & 8 \\
Pooideae & 4 & 9 & 16 \\
TOTAL & 16 & 44 & 86 \\
\hline
\end{tabular}




\section{Subfamilia Bambusoideae}

Plantas perennes, herbáceas o leñosas, rizomatosas; de culmos huecos o sólidos. Láminas foliares planas, anchas, lanceoladas, articuladas con la vaina, presentan un pseudopecíolo. Espiguillas uni a multifloras. Las flores son hermafroditas o unisexuales monoicas, con 3 6 estambres, raramente más. El gineceo con 1 - 3 estilos unidos en la base. Las bambusoideas herbáceas florecen cada año, no así las leñosas que lo realizan en ciclos de muchos años.

Se les encuentran de preferencia en regiones de selvas tropicales y subtropicales húmedas. En el mundo las bambusoideas agrupan a 91 géneros y un número aún no determinado de especies. En el Perú esta familia esta representada por 18 géneros y 67 especies, que habitan mayormente en el bosque montano oriental y en la llanura amazónica. El presente trabajo reporta 23 especies de la Subfamilia Bambusoideae para el área de estudio (Tabla 2)

\section{Clave de géneros de la Subfamilia Bambusoideae}

\section{Bambúes leñosos}

1a. Cañas no ramificadas en el estado vegetativo; vainas y láminas foliares no bien diferenciadas; láminas grandes, de hasta $2 \mathrm{~m}$ de largo.

Neurolepis

1b. Cañas ramificadas en el estado vegetativo; vainas y láminas bien diferenciadas; láminas 3,5-33 cm.

2a. Caña sólida, sin lúmen central, salvo algunas especies con lúmen poco desarrollado.

Chusquea

2b. Caña hueca, con lúmen central, salvo algunas especies con lúmen poco desarrollado. gados.

3a. Cañas moderadamente leñosas, entrenudos del3b. Cañas notoriamente leñosas, entrenudos engrosados.

Rhipidocladum

\section{Bambúes herbáceos}

1a. Nervaduras de la lámina foliar no paralelas a la nervadura central, sino divergentes a ella; hojas largamente pseudopecioladas; inflorescencia en panoja desarticulable y caediza en secciones de varias ramas.

Pharus

1b. Nervaduras de la lámina foliar paralelas a la nervadura central; hojas cortamente pseudopecioladas.

2a. Inflorescencia en espiga comúnmente desarticulable y caediza en segmentos; setas comúnmente desarrolladas.

Pariana

2b. Inflorescencia en panoja o racimo; setas ausentes. Olyra

Tabla 2. Lista de especies y ubicación taxonómica de las Bambusoideas registradas para el Parque Nacional Yanachaga-Chemillén.

\section{Subfamilia I. Bambusoideae}

\section{Tribu: Bambuseae}

\section{Subtribu: Arthrostylidinae}

1. Aulonemia humillima (Pilg.) McClure

2. Aulonemia haenkei (Rup.) McClure

3. Aulonemia queko Goudot

4. Rhipidocladum harmonicum (Parodi) Mc Clure

\section{Subtribu: Chusqueineae}

5. Chusquea picta Pilg.

6. Chusquea tessellata Munro

7. Chusquea depauperata Pilg.

8. Chusquea smithii L.G. Clark

9. Chusquea tarmensis Pilg.

10. Chusquea peruviana E. G.Camus

11. Chusquea scandens Kunth

Subtribu Neurolepidinae

12. Neurolepis aristata Hitch.

\section{Tribu Olyreae}

13. Olyra ecaudata Doell

14. Olyra fasciculata Trin.

15. Olyra loretensis Mez

16. Olyrajuruana $\mathrm{Mez}$

17. Olyra glaberrima Raddi

\section{Tribu Phareae}

18. Pharus virescens Doell

19. Pharus lappulaceus Aubl.

\section{Tribu Parineae}

20. Pariana gracilis Doell

21. Pariana bicolor Tutin

22. Pariana aurita Swallen

23. Pariana radiciflora Sagot ex Doell 


\section{Tribu Bambuseae}

\section{Género Aulonemia Goudot}

Bambú cespitoso rizomatoso, de poca altura, subleñoso. Caña hueca o sólida; vainas foliares con el ápice rodeado comúnmente de setas bien desarrolladas.

A nivel mundial este género tiene 30 especies; para la flora peruana se registran cuatro; tres de las cuales se encuentran en la zona estudiada. Se distribuye desde México, a lo largo de los Andes, hasta Perú y Bolivia; algunas especies se encuentran al oriente de las Guyanas y sur y centro de Brasil ( Clark y Londoño, 1990).

\section{Clave de especies del género Aulonemia}

1a. Planta pequeña, 50-70 cm de largo, caña delgada, menor de dos centímetros de diámetro.

2a. Espiguillas angostas de 0,5-1 mm de ancho; lemas fértiles, de 4,4 a 4,6 mm de largo. A. humillima

2b. Espiguillas de 2-5 mm de ancho; lemas fértiles, de 7 a $8 \mathrm{~mm}$ de largo.

A. haenkei

1b. Planta grande de 3-5 m de largo, caña gruesa de 3$4 \mathrm{~cm}$ de diámetro.

A. queko

\section{Aulonemia humillima (Pilg.) McClure}

El bosque premontano oriental o pluviselva andina siempre verde es el hábitat de este género. Endémica del Perú en los departamentos Amazonas, Loreto, Junín y Pasco, entre los $900-2000 \mathrm{~m}$.

Material estudiado: Provincia de Oxapampa, Cordillera de San Matías, 910 m, D. N. Smith 2045 (MO)

\section{Aulonemia haenkei (Rupr.) McClure}

Habita en el bosque montano oriental y del Perú en Amazonas, Cusco, Huánuco, Huancavelica y Pasco.

Material estudiado: Provincia de Oxapampa, Cordillera Yanachaga, Río San Daniel, 2700 m, D. N. Smith 7666 (USM).

\section{Aulonemia queko Goudot}

Habita en el bosque montano oriental, entre los 2000-3000 m y se distribuye en los ni- veles medios de los Andes de Colombia, Ecuador, Perú y Brasil. En el Perú se ha registrado en los departamentos de Huánuco y Pasco.

Material estudiado: Provincia de Oxapampa, 2700 m. D. N. Smith 7805 (USM).

\section{Género Rhipidocladum McClure}

Bambú cespitoso, perenne, cañas leñosas, erguidas, huecas, con entrenudos alargados de 35 cm o más, con numerosas ramas en los nudos, con ramas complementarias flabeladas, que nacen en un espacio triangular por encima de los nudos.

Este género presenta 12 especies a nivel mundial, dos especies para Perú y una para la zona estudiada.

\section{Rhipidocladum harmonicum (Parodi) McClure}

Habita en el bosque montano oriental y se distribuyen en Ecuador, Perú y Bolivia entre los $2000-3000 \mathrm{~m}$.

En el Perú se ha registrado en los departamentos de Cusco, Junín y Pasco. Los entrenudos de cerca de $1 \mathrm{~m}$ de largo se utilizan para la confección de instrumentos musicales como quena y zampoña y en otras partes del Perú como material de construcciones rústicas.

Material estudiado: Provincia de Oxapampa, San Alberto, 2300 - 2400 m, D. N. Smith y A. Pretel 7644; San Alberto, 2400 m, M. I. La Torre 1164 (USM).

\section{Género Chusquea Kunth}

Plantas rizomatosas con cañas erguidas, tiesas a menudo, péndulas superiormente, entrenudos comúnmente sólidos. Nudos de la parte media de la caña con una yema central de mayor tamaño, flanqueada de varias yemas pequeñas, en la axila de una vaina. El género tiene aproximadamente 130 especies a nivel mundial por lo menos con 12 especies para el Perú y seis especies para la zona de 
estudio. Se le encuentra desde México hasta Argentina y Chile (Renvoize, 1998), ocupando los llamados bosques de neblina o Ceja de la Montaña en el Perú.

\section{Clave de especies del género Chusquea}

1a. Espiguillas de 10-15,3 mm de longitud. Ch. picta

1b. Espiguillas de 5,5-9,5 mm de longitud.

2a. Panícula densa hasta espiciforme.

3a. Glumas de 1-1,5 mm de largo, pubescentes.

Ch. tessellata

3b. Glumas 0,2-0,4 mm de largo, glabras. bozo.

4a. Espiguilla glabra; glumas reducidas a sólo un eslargo.

4b. Espiguilla pubescente; glumas de 0,2-0,4 mm de

2b. Panícula laxa.

5a. Espiguillas de 9-9,5 $\mathrm{mm}$ de largo.

Ch.tarmensis

5b. Espiguillas de 5,3-7 mm de largo.

6a. Láminas foliares de las ramificaciones de 2-4 mm de ancho. Ch. peruviana

6b. Láminas foliares de las ramificaciones de 5-12 mm de ancho.

Ch. scandens

\section{Chusquea picta Pilg.}

El bosque montano oriental es su hábitat y se distribuye en los Andes centrales del Perú y se ha registrado en los departamentos de Huánuco y San Martín.

Material estudiado: Provinicia de Oxapampa, Los Chacos 2550 m, D. Smith y A. Pretel 1515; Santa Bárbara 3100-3200 m, M.I. La Torre, 1617(USM).

\section{Chusquea tessellata Munro}

Se le encuentra en el bosque montano oriental y puna húmeda con presencia de arbustos pequeños y herbazal bajo. Se distribuye en Colombia, Ecuador, Perú y Bolivia. En el Perú se ha registrado en los departamentos de Amazonas, Cusco, Junín, Madre de Dios y Puno.

Material estudiado: Provincia de Oxapampa, Santa Bárbara, 3300 - 3420 m,
D. N. Smith 8106; Santa Bárbara, 3100 3300m, M. I. La Torre 1547 (USM).

\section{Chusquea depauperata Pilg.}

Se les encuentra en el bosque montano oriental y puna húmeda y se distribuye en los Andes centrales del Perú y se ha registrado en los departamentos de Cusco, Huánuco, La Libertad y Pasco.

Material estudiado: Provincia de Oxapampa, Santa Bárbara, D. N. Smith 8106 (MO).

\section{Chusquea smithii L.G. Clark.}

Esta especie habita en el pajonal en los bordes del bosque montano oriental y es conocida sólo de la cordillera Yanachaga.

Material estudiado: Provincia de Oxapampa, Cordillera Yanachaga vía río San Daniel, 3350 - 3420 m, D. N. Smith 7730 (MO).

\section{Chusquea tarmensis Pilg.}

Habita en el bosque montano oriental (bosque tropical húmedo o Ceja de Montaña) entre los $2100-2800 \mathrm{~m}$. Es conocida solamente en los Andes peruanos. Se ha registrado en los departamentos de Junín y Cusco.

Material estudiado: Provincia de Oxapampa, San Gotardo, 2850 m, D. N. Smith 3222 (USM).

\section{Chusquea peruviana E. G. Camus}

Habita en el bosque montano oriental y se distribuye en Ecuador, Perú y Bolivia. En el Perú se ha registrado de los departamentos de Cusco, Puno, Huánuco y Junín.

Material estudiado: Provincia de Oxapampa, entre Oxapampa y Paucartambo, 2850 m, D. N. Smith 1590 (USM).

\section{Chusquea scandens Kunth}

Habita en el bosque montano oriental y se distribuye en Colombia, Ecuador y Perú. En Perú se ha registrado en los departamentos de Amazonas, Cajamarca, Cusco, Huánuco, 
Lambayeque, Pasco, Piura, Puno, San Martín y Huancavelica. La caña se emplea como armazón de techos.

Material estudiado: Provincia de Oxapampa, Cordillera Yanachaga, 2800 m, D. N. Smith 7675; San Alberto, 2650 m, M. I. La Torre 1092; Santa Bárbara, 3300 m, R. Foster 10455 (USM).

\section{Género Neurolepis Meisner}

Plantas herbáceas, erguidas, robustas, leñosas en la base. Cañas simples sin ramificaciones. Láminas de $3-80 \mathrm{~cm}$ de altura por 2 - $6 \mathrm{~cm}$ de ancho. Género neotropical con 10 especies, una especie para Perú y la zona de estudio; casi todas del páramo húmedo de Trinidad, Venezuela, Perú y Bolivia.

\section{Neurolepis aristata Hitchc.}

Habita en el bosque montano oriental y se distribuyen en Colombia, Ecuador y Perú en los departamentos Amazonas, Cusco y La Libertad.

Material estudiado: Provincia de Oxapampa, Cordillera Yanachaga, 3150-3300 m, D. N. Smith 7713; San Gotardo, 2730 2800 m, D. N. Smith y J. Canne 5891; Santa Bárbara, 3100 m, M. I. La Torre 1707 (USM).

\section{Tribu Olyreae}

\section{Género Olyra Linnaeus}

Plantas monoicas, cañas erguidas. Hojas con pseudopeciolo corto; las láminas aovadolanceoladas. Se conocen 23 especies, de las cuales 10 se encuentran en el Perú y cinco en la zona estudiada. Se extienden desde Florida (EE.UU.), México y las Antillas hasta Argentina.

\section{Clave para diferenciar las especies del genero Olyra}

1a. Antecio femenino punteado-granulado.

2a. Glumas no atenuadas; ramas de la panoja cortas y rígidas. 2b. Glumas muy atenuadas; ramas de la panoja laxas. O. fasciculata

1b. Antecio femenino glabro o pubescente, pero no punteado granulado.

3a. Inflorescencia en cañas reducidas, vistosas.

$$
\text { O. loretensis }
$$

3b. Inflorescencia en cañas con hojas, no vistosas.

4a. Antecio femenino con pelos largos cilíndricos, adpresos en toda la superficie.

O. juruana

4b. Antecio femenino con pelos aplanados solo hacia la base y el ápice.

O. glaberrima

\section{Olyra ecaudata Doell}

Habita en el bosque tropical amazónico o selva baja y se distribuye en Centroamérica, Brasil y Perú. En el Perú se ha registrado en los departamentos de Loreto, Pasco y Ucayali.

Material estudiado: Provincia de Oxapampa, 19 km Oeste de Oxapampa, 2080 m, D. N. Smith 2700 (USM).

\section{Olyra fasciculata Trin.}

Habita en el bosque tropical húmedo, se distribuye en Panamá, Perú, Bolivia, Brasil y noroeste de Argentina. En el Perú se ha registrado de los departamentos de Huánuco, Cusco, Junín, Pasco y San Martín.

Material estudiado: Provincia de Oxapampa, Iscozacín, 350 - 450 m, D. N. Smith 2842 (USM)

\section{Olyra loretensis Mez}

Habita en el bosque tropical amazónico o selva baja y se distribuye en Colombia, Perú y Brasil. En el Perú se ha registrado de los departamentos de Junín, Loreto, Madre de Dios y Pasco.

Material estudiado: Provincia de Oxapampa, Paujil, 300 m, R. Foster 8906; Paujil, 300 m, M. I. La Torre 1029 (USM).

\section{Olyra juruana Mez}

Habita en el bosque tropical amazónico o selva baja y se distribuye en Brasil, Perú y Bolivia. En el Perú se ha registrado de los 
departamentos de Cusco, Loreto, Madre de Dios, Pasco.

Material estudiado: Provincia de Oxapampa, Iscozacín, 500 m, R. Foster 7968 (USM).

\section{Olyra glaberrima Raddi}

Su hábitat es el bosque tropical amazónico o selva baja y se distribuye en México, Guatemala, Honduras, este de Brasil y Perú. En el Perú se ha registrado en los departamentos de Madre de Dios y Pasco.

Material estudiado: Provincia de Oxapampa, Iscozacín, Valle de Palcazú, camino a Villa América, R. Foster 9527 (MO).

\section{Tribu Phareae}

\section{Género Pharus P. Browne}

Planta perenne herbácea; las láminas foliares pueden ser lineares hasta oblongas con pseudopecíolo.

A nivel mundial se conocen siete especies, distribuidas en los trópicos del Nuevo Mundo, cuatro especies para Perú y dos especies para la zona estudiada.

\section{Clave de especies del genero Pharus}

1a. Glumas de la espiguilla femenina herbácea, de color verde.

Ph. virescens

1b. Glumas de la espiguilla femenina endurecida, de color marrón.

Ph. lappulaceus

\section{Pharus virescens Doell}

Su hábitat es el bosque secundario, bosque tropical amazónico o selva baja y está distribuido desde Centroamérica hasta Perú y Brasil. En el Perú se ha registrado en los departamentos de Amazonas, Huánuco, Loreto, Madre de Dios, y Ucayali.

Material estudiado: Provincia de Oxapampa, Cordillera de San Matías, 400 m, D. N. Smith 2077 (USM)

\section{Pharus lappulaceus Aubl.}

Habita en el bosque tropical amazónico o selva baja y se distribuye desde las Indias Occidentales hasta Paraguay. En el Perú se encuentra en la cuenca amazónica; habiéndose registrado en los departamentos de Amazonas, Cajamarca, Huánuco, Loreto, Madre de Dios, Pasco, San Martín y Ucayali.

Material estudiado: Provincia de Oxapampa, Gran Pajonal, 1200-1300 m, D. N. Smith 6587 (USM).

\section{Tribu Parianeae}

\section{Género Pariana Aublet}

Plantas herbáceas. Inflorescencia cilíndrica, espiciforme exerta de la vaina más superior. Distribuidas desde Costa Rica hasta Brasil, Perú y norte de Bolivia. En el Perú se encuentra en la llanura amazónica. Este género cuenta con 30 especies, 10 especies para Perú y cuatro especies para la zona de estudio.

\section{Clave de especies del genero Pariana}

1a. Cañas delgadas, delicadas; inflorescencias de 4,5 a $6 \mathrm{~mm}$ de ancho; láminas foliares generalmente menores de $12 \mathrm{~cm}$ de largo por $25 \mathrm{~mm}$ de ancho. P. gracilis

1b. Cañas algo engrosadas; inflorescencias de 7 a 11 $\mathrm{mm}$ de ancho; láminas foliares generalmente mayores de $13 \mathrm{~cm}$ de largo por $3,5 \mathrm{~cm}$ de ancho.

2a. Láminas foliares glabras en la nervadura central en el haz.

3a. Lígula de 2 a $3 \mathrm{~mm}$ de largo; hojas glaucas en el envés, de hasta $17 \mathrm{~cm}$ de largo.

P. bicolor

3b. Lígula de 5 a $15 \mathrm{~mm}$ de largo, hojas no glaucas en el envés de hasta $23 \mathrm{~cm}$ de largo.

P.aurita

2a. Láminas foliares conspicuamente híspido-pubescente en la nervadura central en el haz. $\quad \boldsymbol{P}$. radiciflora

\section{Pariana gracilis Doell}

Su hábitat es el bosque tropical amazónico o selva baja. Se distribuye en Brasil y Perú, habiéndose registrado en los departamentos de Junín y Pasco.

Material estudiado: Provincia de Oxapampa, Paujil, 500 m, P. Hocking 117 (USM). 


\section{Pariana bicolor Tutin}

Habita en el bosque tropical amazónico o selva baja. Se distribuye en Perú, Ecuador, Brasil y Bolivia.

En el Perú se ha registrado en los departamentos de Cusco, Huánuco, Junín y Pasco.

Material estudiado: Provinicia de Oxapampa, Iscozacín, 500 m, M. I. La Torre 1013 (USM).

\section{Pariana aurita Swallen}

El hábitat de este género es el bosque tropical lluvioso. Se le encuentra en Perú y Bolivia. En el Perú se ha registrado en los departamentos de Loreto y Pasco.

Material estudiado: Prov. Oxapampa, Iscozacín, 510 m, M.I.La Torre 1074 (USM).

\section{Pariana radiciflora Sagot ex Doell}

Habita en el bosque tropical amazónico (selva tropical lluviosa). Se distribuye en las Guyanas, Colombia, Perú y Brasil. En el Perú se ha registrado en los departamentos de Loreto y Pasco.

Material estudiado: Provincia de Oxapampa, Iscozacín 510 m, M. I. La Torre 1013 (USM).

\section{Conclusiones}

En el Parque Nacional YanachagaChemillén se encuentra una alta diversidad de Bambusoideas, registrándose el 35\% del total de las especies de esta subfamilia conocidas para el Perú (Tovar, 1998). Esta subfamilia de distribución tropical (Renvoize, 1998) con siete géneros y 23 especies, ocupa el segundo lugar en diversidad, en la zona de estudio, después de las Panicoideas (32). La Subfamilia Bambusoideae se encuentra predominantemente en selva baja y bosque premontano aunque el género Chusquea tiene un rango mayor de distribución, que incluye el bosque montano y el ecotono con la puna (Clark, 1990, 1991, 1992). Se registra una especie endémica del Parque Yanachaga Chemillén que es Chusquea smithii.
Entre las bambusoideas leñosas, el género Chusquea, con siete especies, es el género que presenta mayor diversidad específica. El género Olyra es el mejor representado entre las bambusoideas herbáceas, con cinco especies

\section{Agradecimientos}

Los autores agradecen el apoyo financiero del Fondo de Desarrollo Universitario (FEDU) de la Universidad Nacional Mayor de San Marcos, del Consejo Nacional de Ciencia y Tecnología (CONCYTEC), The University of Maryland Baltimore County, The Nature Conservancy y la Fundación Peruana para la Conservación de la Naturaleza. Expresamos nuestro reconocimiento a Kenneth R. Young, Blanca León, José Roque y Mónica Arakaki por el apoyo en el trabajo de campo, y/o la revisión del manuscrito. Al Biólogo Pedro Aguilar Delgado por su valioso apoyo logístico brindado, así como al personal del Parque Nacional Yanachaga-Chemillén.

\section{Literatura citada}

Aguilar, P. 1986. Yanachaga-Chemillén. Futuro Parque Nacional en la Selva Central del Perú. Boletín de Lima 45:7-21

Brack, A. 1984. Plan Maestro del Parque Nacional Yanachaga Chemillén USAID / PEPP Informe.

Brack, A. 1986. Ecología de un país complejo. En Gran Geografía del Perú: Naturaleza y Hombre. Volumen II., Manfer-Mejía Baca, España: 175-319

Brako, L. \& J. Zarucchi. 1993. Catalogue of the Flowering Plants and Gymnosperms of Peru. Missouri Botanical Garden, Monographs in Systematic Botany 45: 1-1286.

Burkart, A. 1969. Flora ilustrada de Entre Ríos. Parte II. Gramíneas. Colección Científica del INTA. Tomos II, VI. Argentina.

Cano, A. 1994. Sinopsis de la flora fanerogámica altoandina del Parque Nacional del Manu, Cusco. Universidad Nacional Mayor de San Marcos, Tesis para Optar el Grado de Magister en Botánica, Lima.

Cerrate, E, 1969. Manera de Preparar Plantas para un Herbario Museo de Historia Natural. Serie de Divulgación N \# 1.

Clark, L. G. 1990. Chusquea sect. Longiprophyllae (Poaceae: Bambusoideae) A New Andean Section and New Species. Sistematic Botany, 15(4): pp 617-634. 
Clark, L. G. 1991. A new species and new Sections of Rhipidocladum (Poaceae: Bambusoideae) American Journal of Botany 78(9): 1260-1279.

Clark, L. G. 1992. Chusquea Sect. Swallenochloa (Poaceae: Bambusoideae) and allies in Brazil. Brittonia, 44(4), 1992, pp. 387-422.

Clark, L. G. \& X. Londoño. 1990. Three New Andean Species of Aulonemia. (PoaceaeBambusoideae). Ann. Missouri Botanical Garden 77: 353-358.

Clark, L. G. \& X. Londoño. 1991. Miscellaneous new taxa of bamboo (Poaceae: Bambuseae) from Colombia, Ecuador and México. Nord. J. Bot. Section of tropical taxonomy 11: 323-331. Trop 247.

Clark, L., G. Davidse, R. P. Ellis 1989. Natural Hybridazation in Bamboos: Evidence from Chusquea sect. Swallenochloa(Poaceae: Bambusoideae) National Geografhic Research 5(4): 459-476 (1989).

Clayton, W. D. \& S. A.Renvoize.1986. Genera Graminarum. Grasses of the World. Kew Bulletin Additional, Series 13:1-389.

Lot, E. \& F Chiang.(Compiladores).1986.Manual de Herbario. Consejo Nacional de la Flora de México, México. 1-42 pp.
Nicora, E. G. \& Z. E. Rugolo de Agrasar. 1987. Los géneros de gramíneas de América del Sur. Editorial Hemisferio Sur S. A. Buenos Aires. 1576 pp.

Pacheco, V., S. Solari., E. Vivar., P. Hocking.1994.La riqueza biológica del Parque Nacional Yanachaga Chemillén. Magistri et Doctores 7: 3-6

Pohl, R. 1980. Family No 15, Gramineae Flora Costarricenses. Fieldiana New Series, No 3, Chicago.

Renvoize, S. A. 1998. Gramíneas de Bolivia. The Royal Botanic Gardens, Kew. 644 PP

Tovar, O. 1993. Las Gramíneas (Poaceae) del Perú. Ruizia, Tomo 13, Madrid. 481 pp

Tovar, O. 1998. Diversidad de las gramíneas (Poaceae) del Perú. En Halffter, G. (Compilador), Acta Zoológica Mexicana Nueva Serie, Volumen especial. Pp. 117-131

Weberbauer, A. 1945. El Mundo Vegetal de los Andes Peruanos. Ministerio de Agricultura, Lima.

Womersley, J. 1981. Plant collecting and Herbarium developement. FAO Plant Production and Protection Paper.

Young, K. R. \& N. Valencia. 1992. Introducción: Los bosques Montanos en el Perú. En Young K. R. \& N. Valencia (Eds.), Biogeografía, Ecología y Conservación del Bosque Montano en el Perú. Memorias del Museo de Historia Natural U. N. M. S. M. (Lima) 21: 5-9. 